\title{
Evaluation of metabolomics profiles of grain from maize hybrids derived from near-isogenic GM positive and negative segregant inbreds demonstrates that observed differences cannot be attributed unequivocally to the GM trait
}

\author{
George G. Harrigan ${ }^{1} \cdot$ Tyamagondlu V. Venkatesh $^{1} \cdot$ Mark Leibman $^{2} \cdot$ \\ Jonathan Blankenship ${ }^{3} \cdot$ Timothy Perez $^{3} \cdot$ Steven Halls $^{4} \cdot$ Alexander W. Chassy $^{5}$. \\ Oliver Fiehn ${ }^{5,7} \cdot$ Yun Xu$^{6} \cdot$ Royston Goodacre $^{6}$
}

Received: 10 November 2015/Accepted: 22 February 2016/Published online: 15 March 2016

(C) Springer Science+Business Media New York 2016

\begin{abstract}
Introduction Past studies on plant metabolomes have highlighted the influence of growing environments and varietal differences in variation of levels of metabolites yet there remains continued interest in evaluating the effect of genetic modification (GM).

Objectives Here we test the hypothesis that metabolomics differences in grain from maize hybrids derived from a
\end{abstract}

Electronic supplementary material The online version of this article (doi:10.1007/s11306-016-1017-6) contains supplementary material, which is available to authorized users.

Mark Leibman

mark.e.leibman@monsanto.com

1 Compositional Biology Center, Monsanto Company, St. Louis, MO, USA

2 Regulatory Affairs, Monsanto Company, St. Louis, MO, USA

3 Statistics Technology Center, Monsanto Company, St. Louis, MO, USA

4 Chemistry Technology, Monsanto Company, St. Louis, MO, USA

5 Genome Center - Metabolomics, University of California at Davis, Davis, CA, USA

6 School of Chemistry, Manchester Institute of Biotechnology, University of Manchester, 131 Princess Street,

Manchester M1 7ND, UK

7 Biochemistry Department, King Abdulaziz University, Jeddah 21589, Saudi Arabia series of GM (NK603, herbicide tolerance) inbreds and corresponding negative segregants can arise from residual genetic variation associated with backcrossing and that the effect of insertion of the GM trait is negligible.

Methods Four NK603-positive and negative segregant inbred males were crossed with two different females (testers). The resultant hybrids, as well as conventional comparator hybrids, were then grown at three replicated field sites in Illinois, Minnesota, and Nebraska during the 2013 season. Metabolomics data acquisition using gas chromatographytime of flight-mass spectrometry (GC-TOF-MS) allowed the measurement of 367 unique metabolite features in harvested grain, of which 153 were identified with small molecule standards. Multivariate analyses of these data included multiblock principal component analysis and ANOVA-simultaneous component analysis. Univariate analyses of all 153 identified metabolites was conducted based on significance testing $(\alpha=0.05$ ), effect size evaluation (assessing magnitudes of differences), and variance component analysis.

Results Results demonstrated that the largest effects on metabolomic variation were associated with different growing locations and the female tester. They further demonstrated that differences observed between GM and non-GM comparators, even in stringent tests utilizing nearisogenic positive and negative segregants, can simply reflect minor genomic differences associated with conventional back-crossing practices.

Conclusion The effect of GM on metabolomics variation was determined to be negligible and supports that there is no scientific rationale for prioritizing GM as a source of variation.

Keywords Marker-assisted breeding - Marker-assisted back crossing - Metabolomics - Genetic modification . Natural variability · Genetically modified (GM) traits . Maize (Zea mays) 


\section{Introduction}

Modern agricultural biotechnology provides an efficient and effective way to improve crop varieties and to enable sustainable food production. The benefits conferred by genetically modified (GM) crops have led to their widespread adoption globally, and in 2014 , GM crops were planted by a total of 18 million farmers across 28 countries (James 2014).

An important consideration in the development of new crop varieties is continued nutritional quality and safety in the food chain. New biotechnology-derived traits in GM crops are currently evaluated in safety assessments that follow internationally recognized guidelines and that are accepted by regulatory agencies world-wide (OECD 2006; Codex 2009). These assessments include detailed molecular characterization of a new GM variety to ensure integration of the intended DNA sequence as well as measurement of levels of expressed products. One of the major principles behind current assessments is the concept of substantial equivalence where a conventional variety is used as a comparator for evaluating the phenotypic and nutrient compositional characteristics of the new product. The selected conventional comparator typically shares a similar genetic background to the new product (i.e. is nearisogenic), but does not express the new biotechnologyderived trait. Comparative assessments have demonstrated substantial equivalence between GM crops and their nearisogenic conventional counterparts and that GM has little, if any, effect on pre-existing crop characteristics other than the introduction of intended benefits associated with the new desired traits. Past assessments have thus provided an assurance of safety that has extended over two decades of GM commercialization (EU 2010; Herman and Price 2013; Prado et al. 2014). A recent review of crop composition studies observed that "over the past 20 years, the U.S. FDA found all of the 148 transgenic events that they evaluated to be substantially equivalent to their conventional counterparts, as have the Japanese regulators for 189 submissions." prompting the authors (Herman and Price 2013) to conclude that "compositional equivalence studies uniquely required for GM crops may no longer be justified on the basis of scientific uncertainty."

The consistent demonstration of safety for commercial GM products stems both from the low potential of the technology to induce meaningful unintended genetic changes (see Ladics et al. 2015; Schnell et al. 2015, for comprehensive reviews) that would impact a phenotypic trait, as well as the strict quality guidelines in the selection and development of new biotechnology-derived traits and commercial products (Privalle et al. 2013; Prado et al. 2014).

With respect to crop composition, it is now well-established that conventional breeding, varietal differences, and differences in growing location are much greater sources of compositional variation than GM (Berman et al. 2011; Harrigan et al. 2010; Harrigan and Harrison, 2012; Zhou et al. 2011). It was very recently reported that even "incidental" but well-established features of conventional plant breeding such as residual genetic variation from conventional back-crossing in maize can be more associated with compositional variation than GM (Venkatesh et al. 2015b). The study on which these conclusions were based included assessment of a diverse range of GM traits (herbicide tolerance, insect protection, and drought tolerance) expressed in multiple genetically distinct maize hybrids. The influence of residual genetic variation was further observed even in stringent tests involving GM and non-GM comparators derived from paired positive and negative segregants (Venkatesh et al. 2015b).

Repeated findings of compositional equivalence between GM and non-GM crops, and the greater impact on compositional variation of other factors such as those associated with conventional breeding and growing environment, have not precluded advocacy for expanding analytical requirements for GM assessments (e.g. Davies 2010). The ability of metabolomics to measure a wide range of small molecule metabolites (Goodacre et al. 2004) has prompted suggestions that it may aid in identifying potential unintended differences between GM and non-GM crops that could be attributed to trait insertion (Rischer and Oksman-Caldentey 2006). However, consistent with results from composition studies, metabolomics research has simply reinforced the conclusion that GM trait insertion does not have any meaningful effect on crop metabolite profiles (Ricroch et al. 2011; Ricroch 2013). For example, metabolomic studies conducted on maize grain have, overall, highlighted the dominant effects of conventional germplasm and growing location on metabolite profiles relative to the negligible effect of GM insertion (Asiago et al. 2012; Baniasadi et al. 2014; Frank et al. 2012; Röhlig et al. 2009; Skogerson et al. 2010).

We hypothesized that, as with crop composition, "incidental" features of conventional plant breeding, such as residual genetic variation from back-crossing in maize, would be associated with greater metabolomics variation than GM (Venkatesh et al. 2015a, b). The purpose of the current study was therefore, (i) to compare the metabolomic profiles of grain from maize hybrids derived from paired GM trait-positive and their corresponding traitnegative segregant inbreds, and (ii) to evaluate any observed differences in the context of residual genetic variation associated with the near-isogenic inbreds developed from the same recurrent parent (RP) during successive backcrossing. The experimental design allows a systematic assessment of the influence of residual genetic 
variation associated with back-crossing on metabolomic variation.

In this study, four NK603-positive and negative segregant inbred males were crossed with two different females (testers). The resultant hybrids, as well as conventional comparator hybrids, were grown at three replicated sites in Illinois, Minnesota, and Nebraska during the 2013 growing season. Metabolomics data acquisition using a gas chromatographytime of flight-mass spectrometry (GC-TOF-MS) platform allowed measurement of 367 metabolite features in harvested grain, of which 153 metabolites were fully identified compounds (i.e. identified to level 1 of the Metabolomics Standards Initiative (MSI; Sumner et al. 2007)). Initial multivariate data analyses included multiblock principal component analysis (MB-PCA) and ANOVA-simultaneous component analysis (ASCA) conducted on the entire GC-TOF-MS data set. To probe the relative contributions of residual genetic variation associated with backcrossing (i.e. differences within hybrids derived from the trait-positive and trait-negative segregants) and GM trait effects, a detailed univariate analysis of all 153 identified metabolites was also conducted. This analysis included significance testing ( $\alpha=0.05)$, effect size evaluation (assessing magnitudes of differences), and variance component analysis. The study therefore offers an assessment of whether earlier observations (Venkatesh et al. 2015a, b) that compositional differences between near-iosgenic GM and conventional maize hybrids are associated with backcrossing also extends to metabolomics evaluations, including those involving near-isogenic negative segregants.

\section{Materials and methods}

\subsection{Production and genetic fingerprinting of inbred variants}

Positive and negative inbred variants of NK603 were produced by standard marker-assisted backcrossing (MABC) methods (Eathington et al. 2007) and as described in Venkatesh et al. (2015b) (see Fig. 1 for an overview of backcrossing and Fig. S1 for a schematic of the procedure followed in this study). The variants were fingerprinted using the Illumina (San Diego, CA, USA) Infinium $^{\mathrm{TM}}$ platform. The Infinium ${ }^{\mathrm{TM}}$ platform used for genotyping consisted of 35, 000 SNPs markers. The genotyping analysis is described in detail in Venkatesh et al. (2015a). Table S1 shows the genetic similarity of the inbred lines used in this study to the recurrent parent.

\subsection{Hybrid production}

Hybrid seed production was similar to that described in Venkatesh et al. (2015b). The 18 hybrid entries are listed in
Table 1 and were generated as follows; the testers, T2052Z and S4062Y, were crossed to, (i) four traited inbred variants (positive segregants, POS), (ii) four null inbred variants (negative segregants, NEG), and (iii) the control recurrent parent (RP), R8190.

Field Trials The hybrids were planted in the field during the 2013 growing season using a randomized complete block design at three different locations in U.S. (Steele County, Minnesota [MNOW]; Polk County, Nebraska (NEST]; and Warren County, Illinois [ILMN]). At each location, the plots were comprised of four rows $(7$ meters long and 0.7 meters between rows) and each plot was planted in three replications (blocks). Maize grain was hand-harvested. Dried ears from each row were bulkshelled and grain was stored at room temperature before shipping to Monsanto Company in St. Louis, MO. Grain samples were homogenized by grinding on dry ice to a fine powder and stored frozen at approximately $-20^{\circ} \mathrm{C}$. One sample from the ILMN location (one replicate of POS T1B1 with tester T2052Z) and one sample from the NEST location (one replicate of NEG T1A2 with tester T2052Z) were lost during collection and processing.

\subsection{GC-MS sample extraction, derivatization, and profiling}

Powdered grain samples (20 mg) were extracted with $1 \mathrm{~mL}$ of degassed extraction solvent (5:2:2 methanol:chloroform:water). The mixture was vortexed for $6 \mathrm{~min}$ at $4{ }^{\circ} \mathrm{C}$ prior to centrifugation at $14,000 \mathrm{x} g$ for $2 \mathrm{~min}$. The supernatant $(200 \mu \mathrm{L})$ was dried under vacuum at room temperature. Derivatization was performed as previously described (Fiehn et al. 2008) with $10 \mu \mathrm{L}$ of methyloxamine hydrochloride (40 $\mathrm{mg} / \mathrm{mL}$ in pyridine) added to each sample prior to shaking at $30{ }^{\circ} \mathrm{C}$ for $1.5 \mathrm{~h}$ followed by the addition of a mixture $(91 \mu \mathrm{L})$ of MSTFA containing fatty acid methyl ester (FAME) markers into the sample and incubated at $37{ }^{\circ} \mathrm{C}$ for $30 \mathrm{~min}$.

Samples were analyzed using a GC-TOF-MS approach (Fiehn et al. 2008; Kind et al. 2009). The study design was entered into the MiniX database (Kind et al. 2009). A Gerstel MPS2 automatic liner exchange system (ALEX) was used to eliminate cross-contamination from sample matrix occurring between sample runs. Derivatized sample injections of $0.5 \mu \mathrm{L}$ were made in splitless mode with a purge time of $25 \mathrm{~s}$ and temperature program as follows: 50 to $275^{\circ} \mathrm{C}$ at $12{ }^{\circ} \mathrm{C} / \mathrm{s}$ and held for $3 \mathrm{~min}$. An Agilent 6890 gas chromatograph (Santa Clara, CA) was used with a $30 \mathrm{~m}$ long, $0.25 \mathrm{~mm}$ i.d. Rtx5Sil-MS column with $0.25 \mu \mathrm{m}$ $5 \%$ diphenyl film; an additional $10 \mathrm{~m}$ integrated guard column was used (Restek, Bellefonte PA). Chromatography was performed at a constant flow of $1 \mathrm{~mL} / \mathrm{min}$, 


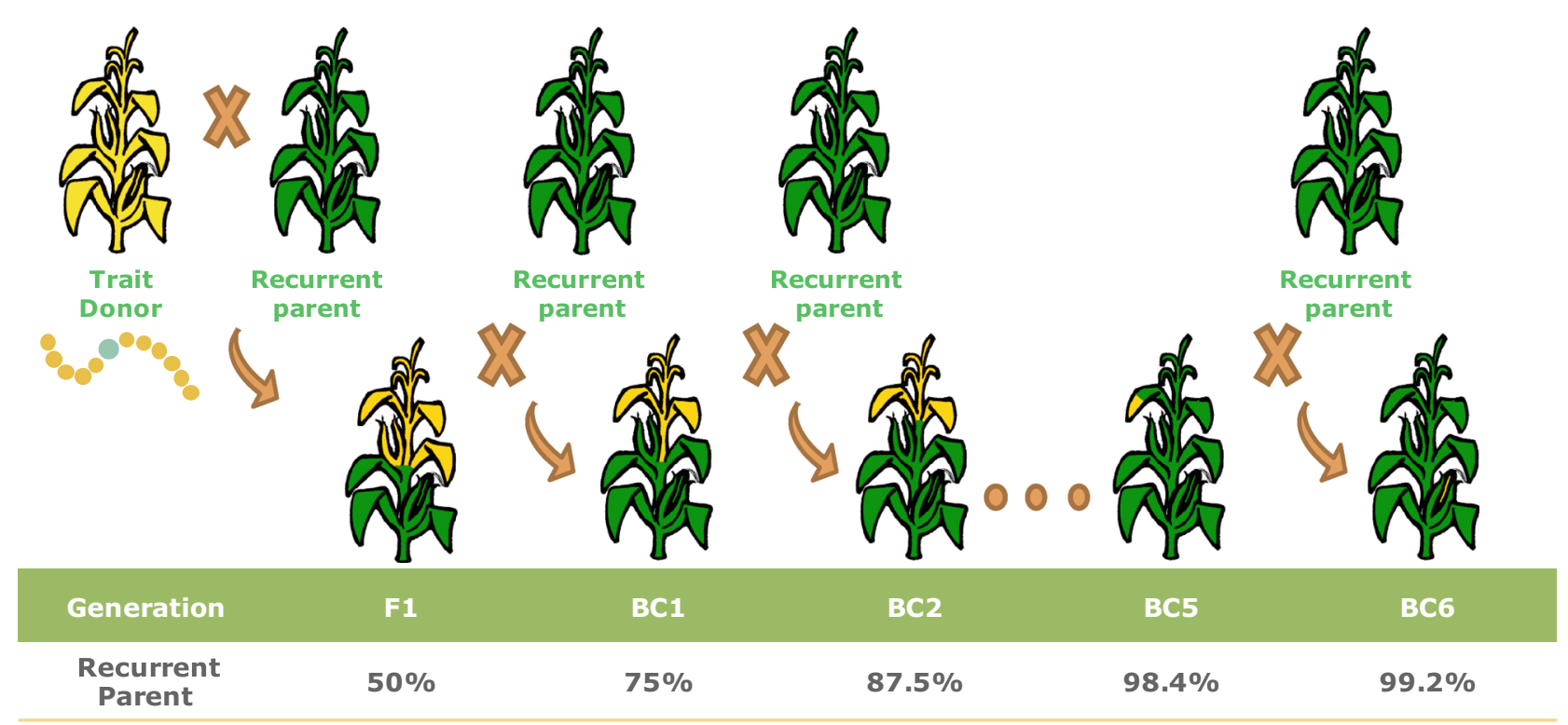

Fig. 1 In plant breeding, selected individuals are crossed to introduce or combine desired trait characteristics into new offspring; this necessitates numerous generations of backcrossing to establish the desired trait characteristics fully. Each successive backcross increases the genetic similarity of the new offspring to the recurrent parent, e.g. $75 \%$ similar at $\mathrm{BC}_{1}$ through to $99.2 \%$ by $\mathrm{BC}_{6}$. These numbers are based on how much of the recurrent parent genome can be

ramping the oven temperature from 50 to $330{ }^{\circ} \mathrm{C}$ over $22 \mathrm{~min}$. Mass spectrometry used a Leco Pegasus IV time of flight mass (TOF) spectrometer with $280{ }^{\circ} \mathrm{C}$ transfer line temperature, electron ionization at $-70 \mathrm{eV}$ and an ion source temperature of $250{ }^{\circ} \mathrm{C}$. Mass spectra were acquired from $\mathrm{m} / \mathrm{z} 85-500$ at $20 \mathrm{spectra} / \mathrm{s}$ and $1750 \mathrm{~V}$ detector voltage.

Result files were exported to servers and further processed using the metabolomics BinBase database (Kind et al. 2009). All database entries in BinBase were matched against the Fiehn mass spectral library of 1200 authentic metabolite spectra using retention index and mass spectrum information or the NIST11 commercial library. Identified metabolites were reported if present with at least $50 \%$ of the samples per study design group (as defined in the MiniX database).

Data from samples were exported to the netCDF format for further data evaluation with BinBase. Briefly, output results were exported to the BinBase database and filtered by multiple parameters to exclude noisy or inconsistent peaks. Quantification was reported as peak height using the unique ion as default. Result files were transformed by calculating the sum intensities of all structurally identified compounds for each sample and then dividing all data associated with a sample by the corresponding metabolite sum. Data are presented in Supplementary File S1. theoretically regained at each step; however slight variations can occur. Marker-assisted methodologies that utilize DNA markers to enable selection of plant individuals that contain the greatest number of favorable alleles can reduce the number of generations required to get close to $99 \%$ similarity as adopted in the generation of the inbred variants of this study (Fig. S2)

\subsection{Multivariate statistical analysis}

Descriptions of both multiblock-principal component analysis (MB-PCA) and ANOVA-simultaneous component analysis (ASCA) are described in detail elsewhere (Smilde et al. 2003, 2005; Xu and Goodacre 2012; Xu et al. 2014; Zwanenburg et al. 2011). A brief overview of the analyses is presented below to facilitate data interpretation.

For MB-PCA, the original metabolite data (Supplementary File S1) were repartitioned into a series of blocks according to the experiment design (i.e., blocking). Through such blocking, one particular factor becomes a baseline and no longer has an effect on the data, whereas the other factor(s) become a common trend across all of the blocks making their influence more apparent. This approach facilitates analysis of systems with multiple and potentially interfering factors. MB-PCA generates three types of results: (1) super scores, which show common trends across all the blocks; (2) block scores, which show individual patterns of the specific blocks; and (3) block loadings, which contain the variable contributions to the MB-PCA model.

ASCA is another recently proposed model aimed at the analysis of data with multiple factors. In ASCA, the data matrix is decomposed into the sum of a series of effect matrices and each effect matrix contains the level averages 
Table 1 Overview of entries associated with the hybrid sets

\begin{tabular}{lll}
\hline Female Inbred & Male Inbred & Control \\
\hline T2052Z & RP & \\
T2052Z & POS A1 \\
T2052Z & POS A2 & \\
T2052Z & NEG A1 & \\
T2052Z & NEG A2 & \\
T2052Z & POS B1 & Control \\
T2052Z & POS B2 \\
T2052Z & NEG B1 \\
T2052Z & NEG B2 \\
S4062Y & RP \\
S4062Y & POS A1 \\
S4062Y & POS A2 \\
S4062Y & NEG A1 \\
S4062Y & NEG A2 \\
S4062Y & POS B1 \\
S4062Y & POS B2 \\
S4062Y & NEG B1 \\
S4062Y & NEG B2 & \\
\hline
\end{tabular}

The entries for this study were based on the listing in Table. A total of four paired positive and negative segregants (e.g. POS A1 and NEG A1) were generated through backcrossing as shown in Fig 2. Each segregant as well as the recurrent parent (RP) was crossed with two different testers for a total of nine (4 POS, 4 NEG, one RP) hybrid entries per tester set

for each factor, which represent the effect of that particular factor. The variations that cannot be explained by the model are put into the residual matrix $\boldsymbol{\varepsilon}$. In this study there are three factors of interest, and thus the ASCA model is written as

$$
\begin{aligned}
X= & \mathbf{1} \times m^{\mathrm{T}}+X_{\mathrm{i}}+X_{1}+X_{\mathrm{s}}+X_{(\mathrm{il})}+X_{(\mathrm{is})}+X_{(\mathrm{ls})}+X_{(\mathrm{ils})} \\
& +\varepsilon
\end{aligned}
$$

where $\boldsymbol{m}^{\boldsymbol{T}}$ is the mean vector of the full matrix; $\boldsymbol{l}$ is a column vector of ones; $\boldsymbol{X}_{\mathrm{i}}, \boldsymbol{X}_{1}$ and $\boldsymbol{X}_{\mathrm{s}}$ are the effect matrices of the three factors, location, trait, and tester, respectively. $\boldsymbol{X}_{\text {(il) }}, \boldsymbol{X}_{(\mathrm{is})}, \boldsymbol{X}_{(\mathrm{ls})}$, and $\boldsymbol{X}_{\text {(ils) }}$ are the interaction matrices between these factors, respectively. PCA was performed on each effect matrix, respectively, to calculate loadings, and the score of each effect matrix was obtained by adding the residual matrix $\boldsymbol{\varepsilon}$ back to the effect matrix and projected into the PC space via the corresponding loadings. For example, the score of the effect matrix of the location factor $\boldsymbol{T}_{\mathrm{i}}$ is calculated as the following:

$X_{\mathrm{i}}=\left(\Gamma_{\mathrm{i}}\right) \times P_{\mathrm{i}}^{\mathrm{T}}$

where $\boldsymbol{\Gamma}_{\mathrm{i}}$ and $\boldsymbol{P}_{\mathrm{i}}$ are the scores and loadings matrix obtained by PCA performed on $\boldsymbol{X}_{\boldsymbol{i}}$.
$T_{\mathbf{i}}=\left(X_{\mathbf{i}}+\varepsilon\right) \times P_{\mathbf{i}}$

Unlike MB-PCA, ASCA is essentially a supervised technique; thus, an appropriate validation procedure is needed. In this paper, we employed a permutation testbased validation procedure proposed by Zwanenburg et al. (2011). In the validation, the magnitude of the effect of the factor of interest is expressed as the sum of squares (SSQs) of $\boldsymbol{\Gamma}$. A total number of $n$ permutations (in this study $n=1000)$ are performed, and in each permutation the labels of the samples are randomly permutated, ASCA is performed on the data using the permuted label, and the SSQs of $\Gamma$ are recorded. All of the SSQs of $\Gamma$ form a null distribution, and the SSQ of $\Gamma$ calculated from the ASCA model using the known labels (i.e., the observed SSQ) is then compared with the null distribution. An empirical $p$ value can then be derived by counting the number of permutations that obtained equal or higher SSQs than the observed SSQ.

Metabolite data were autoscaled so that each variable had zero mean and unit standard deviation before being subjected to MB-PCA and ASCA. All of the multivariate analyses were conducted using in-house scripts written in MATLAB R2012a environment (Mathworks, Natick, MA, USA).

\subsection{Univariate statistical analysis}

Univariate statistical analyses were performed using SAS Software (SAS. 2012 Software Release 9.4 (TS1M1). Copyright 2002-2012 by SAS Institute Inc., Cary, NC).

All identified metabolites were statistically analysed using a mixed model analysis of variance, unidentified metabolites were excluded from analysis. A combined-site analysis was performed using the SAS procedure PROC MIXED to fit the following model:

$$
\begin{aligned}
\mathrm{Y}_{\mathrm{ijk} k \mathrm{mn}}= & \mathrm{U}+\mathrm{L}_{\mathrm{i}}+\mathrm{R}(\mathrm{L})_{\mathrm{j}(\mathrm{i})}+\mathrm{T}_{\mathrm{k}} \\
& +\mathrm{V}(\mathrm{T})_{\mathrm{l}(\mathrm{k})}+\mathrm{S}(\mathrm{VT})_{\mathrm{m}(\mathrm{k})}+\mathrm{LE}_{\mathrm{in}}+\mathrm{e}_{\mathrm{ijk} k \mathrm{mn}}
\end{aligned}
$$

where $Y_{\mathrm{ijklmn}}$ is the unique individual observation, $\mathrm{U}$ is the overall mean, $\mathrm{L}_{\mathrm{i}}$ is the random location effect, $\mathrm{R}(\mathrm{L})_{\mathrm{j}(\mathrm{i})}$ is the random replicate within location effect, $T_{k}$ is the Tester effect, $V(T)_{1(k)}$ is the Variant within Tester effect, $\mathrm{S}(\mathrm{VT})_{\mathrm{m}(\mathrm{kk})}$ is the Segregant within Variant and Tester combination effect, and $\mathrm{LE}_{\mathrm{in}}$ is the random site by entry interaction effect, and $\mathrm{e}_{\mathrm{ijklmn}}$ is the residual error.

A residual is the difference between the observed value and its predicted value from a statistical model. A studentized residual is scaled so that the residual values tend to have a standard normal distribution when outliers are absent. Thus, most values are expected to be between \pm 3 . Extreme data points that are also outside of the \pm 6 studentized residual range are considered for exclusion, as 
outliers, from the final analyses. We chose to employ outlier exclusion because in doing so we would be more conservative and more likely to error in favor of identifying more potential differences rather than fewer differences. A total of 16 observations had studentized residuals outside of the \pm 6 range (See Supplementary File S2 for outlier information). All but the observations for 2-4-diaminobutyric acid, $N$-methlalanine, and ornothine were identified as outliers and removed from analysis. A further outlier test resulted in a further five observations being removed as shown in Supplementary File S2.

For each metabolite, the following comparisons were conducted within each tester set (and shown in schematic form in Supplementary Information.),

Comparison 1 The combined-site mean of the control hybrid [i.e. the hybrid derived from the recurrent parent (RP)] was compared to the combined-site mean of all the hybrids derived from each of the negative and positive segregant inbreds (Fig. S2).

Comparison 2 The combined-site mean for each traitpositive hybrid was compared to the paired negative segregant-derived hybrids, i.e. POS A1 was compared to NEG A1, POS A2 was compared to NEG A2, and so on (Fig. S3).

Statistically significant differences between the mean values were declared at $\alpha=0.05$ (Table 2).

Further pairwise comparisons within each tester set were conducted (Supplementary Files S2 and S3) as described below and as shown in schematic form in Figs.S 4 and S5.

Comparison 3 The combined-site means for each traitpositive hybrid were compared to each other i.e. POS A1 was compared to each of POS A2, POS B1, and POS B2
Table 2 Significant differences $(\alpha=0.05)$ between hybrid entries

\begin{tabular}{llll}
\hline Comparison & Significant & Count & $\%$ \\
\hline Control vs. NEG & No & 1179 & 96.32 \\
Control vs. NEG & Yes & 45 & 3.68 \\
Control vs. POS & No & 1176 & 96.08 \\
Control vs. POS & Yes & 48 & 3.92 \\
POS vs. NEG & No & 1182 & 96.57 \\
POS vs. NEG & Yes & 42 & 3.43 \\
\hline
\end{tabular}

The number of comparisons is the number of metabolite components $(153) \times$ testers $(2) \times$ comparions within each set (4) (i.e., for control vs NEG) there were four comparisons for each metabolite, for control versus POS there were four comparisons, and for POS versus NEG 4 there were comparions (paired). This is shown in schematic form in Figs. S1 and S2

comparisons between POS and NEG variants were made. In other words, POS A1 was compared to NEG A2, NEG B1, and NEG B2. POS A2 ws compared to NEG A1, NEG $\mathrm{B} 1$, and NEG B2. Analogous comparisons were made for POS B1 and POS B2.

Arithmetic means were used to assess the magnitudes of differences observed between the comparator hybrids for all identified metabolites using the following approach. Firstly, the individual site mean for each component was calculated for each hybrid entry (whether trait-positive, trait-negative, or control) at all sites. Secondly, for each tester, the combined-site mean of each metabolite was calculated for the conventional control. Each individual site hybrid entry mean (whether trait-positive, trait-negative, or control) was then expressed as the percent difference from the combined-site conventional control mean as represented below.

$\frac{\text { Individual site mean }(\text { control }) \text { minus combined site mean }(\text { control }) \times 100}{\text { Combined site mean }(\text { control })} \times 100$

$\frac{\text { Individual site mean (POS) minus combined site mean (control) }}{\text { Combined site mean (control) }} \times 100$

Individual site mean (NEG) minusCombined site mean (control)

Combined site mean (control) $\times 100$

(Fig. S3), POS A2 was further compared to POS B1 and to POS B2, and finally POS B1 was compared to POS B2. This pairwise scheme was replicated for the hybrids generated from the negative segregants inbreds.

Comparison $4 \mathrm{In}$ addition to the paired POS-NEG comparisons of the combined-site means (POS A1 to NEG A1 and so on) within Comparison 2 all remaining possible
This allowed a distribution of magnitudes of differences to be gernerated for the control hybrids, the POS hybrids and the NEG hybrids. Histograms of these distributions for each individual metabolite were subsequently generated.

Variance components analysis (VCA) was also conducted to estimate the relative contribution of the experimental factors to the total variance in the study. In this 


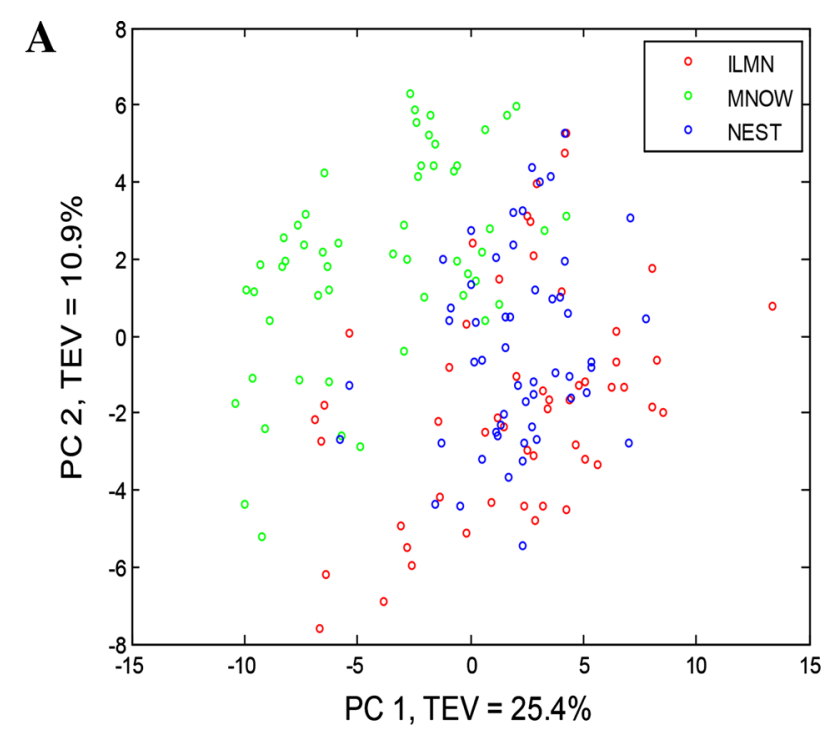

\section{B MB-PCA, super scores plot (location blocking)}
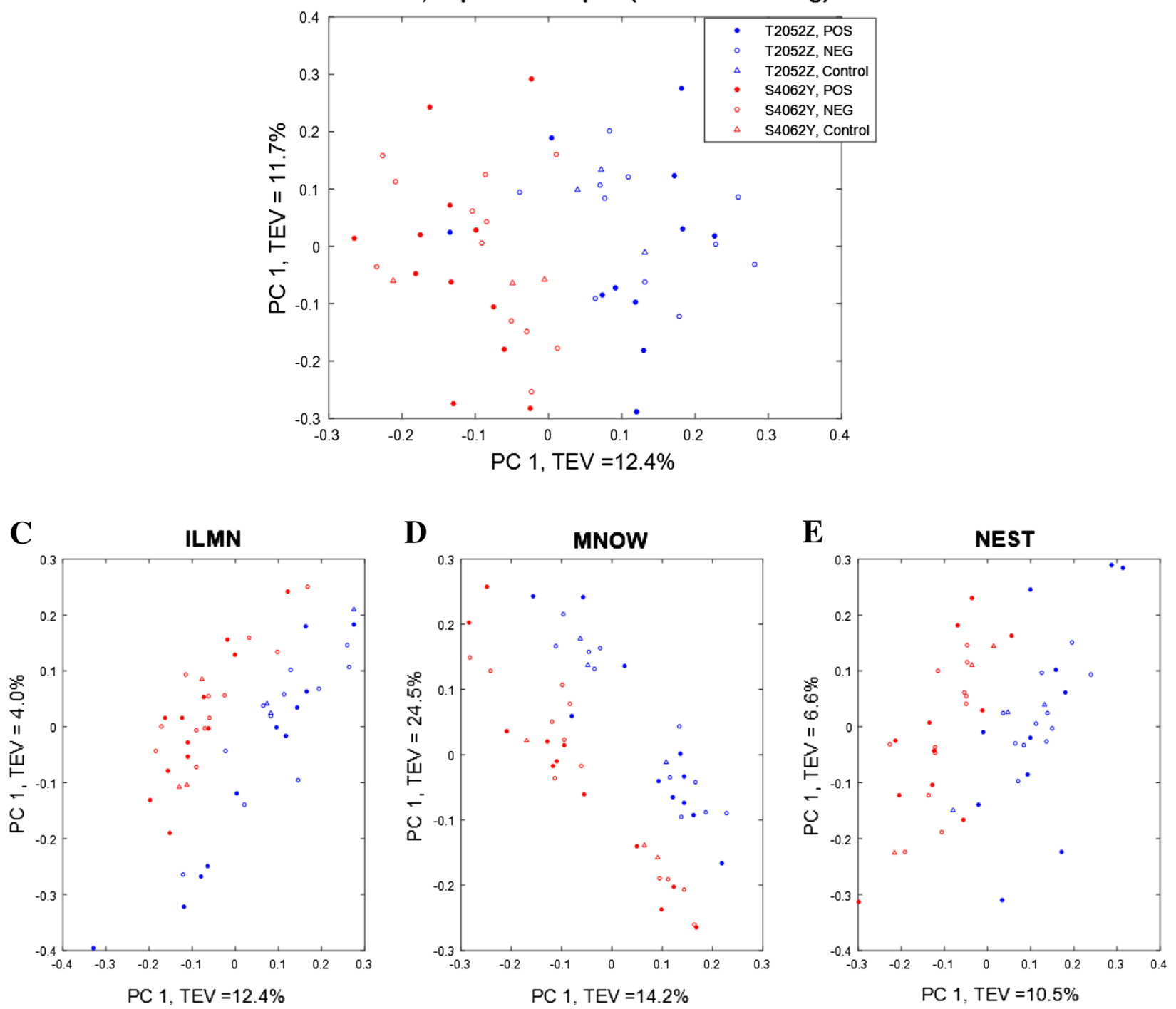
4Fig. 2 PCA and MB-PCA of maize grain metabolome data. PCA showed the effect of location (Fig. 2a). MB-PCA showed that the female tester had a significant influence on the data at each location as can be seen in the super scores (Fig. 2b). The block scores (Fig. 2c-e) showed similar discrimination at all three locations [Illinois (ILMN)], Minnesota (MNOW), Nebraska, (NEST). No clear separation between the GM and non-GM hybrids was observed. TEV total explained variance

application, all effects from the combined-site ANOVA model were set as random effects. The SAS procedure PROC MIXED was employed to run the analysis. The output table of covariance parameter estimates from SAS PROC MIXED procedure gives estimates of the variance component parameters for each of model components. The variance component parameters of each model component were divided by the total variance to obtain the variance proportions for each metabolite.

\section{Results and discussion}

\subsection{Genetic characterization of inbred germplasm}

The design of the experiment was based on generating multiple genetically similar positive (POS) and negative (NEG) segregant male variants that would subsequently be used in hybrid production. A schematic for the generation of the eight inbred variants (four POS and four NEG) is shown in Fig. 1. The genetic similarity of these inbred lines to the corresponding conventional line (recurrent parent) as well as to each other, was determined as described in materials and methods and, as calculated, ranged from 94 to $>99 \%$. Results of the genetic similarity analysis are presented in Table S1. The observed minor differences in the genetic profiles of the inbreds allow testing of whether "near-isogenic effects" can contribute to potential differences between GM and nonGM comparator hybrids derived from these inbreds (Venkatesh et al. 2015a, b).

\subsection{Metabolomics data acquisition}

As described in materials and methods, the genetically characterized male inbred variants were crossed with two different female testers (Table 1) to generate hybrids which were subsequently grown at multiple locations in the US. GC-MS based profiling of the harvested mature grain allowed measurement of 367 metabolite features of which 153 were identified to level 1 of the MSI. These 153 metabolites encompassed a diverse range of biosynthetic and biochemical classes (see Supplementary File S1 for all metabolite data). Supplementary Files S3 and S4 contain a summary of least square means for identified metabolites for the hybrids associated with the S4062Y and T2052Z testers, respectively.

\subsection{Multivariate analysis}

As part of an initial exploratory assessment, principal components analysis (PCA), one of the most popular tools for visualizing metabolomics data, was conducted on the full data matrix. Results highlighted the predominant effect of growing location on variation in this full metabolome data set (Fig. 2a). In order to better discern the effects of other factors (tester, near-isogenic, GM) of interest in this study we subsequently used two different PCA variants, multi-block (MB)-PCA and ANOVA-simultaneous component analysis (ASCA).

\subsection{Multiblock (MB)-PCA}

A series of MB-PCA models was created by repartitioning (i.e. blocking) the data so that the dominant factor on metabolomic variation as established from the original PCA, (that is to say, location) became a background and the potential effect of other factors of interest could be assessed across all location blocks. The scores plots from the MB-PCA model are shown in Fig. 2b-e. These clearly show that, at each different location, the female tester had a significant influence on metabolomic variation. This can be seen in the super scores (Fig. 2b); the block scores (Figs. 2c-e) showed similar discrimination for all three locations. No clear separation between the GM and nonGM hybrids was observed in the scores plots of this model.

\subsubsection{ANOVA-simultaneous component analysis (ASCA)}

The original PCA highlighted that growing location had the greatest impact on the maize metabolome when assessing all metabolite features while MB-PCA established that use of a different female tester to generate the maize hybrids also had an effect. The clustering from both of these algorithms indicated that there was no discernible effect of GM or of residual genetic variation. We tested this observation by employing an ASCA model. In ASCA, as described in materials and methods, the data matrix is modeled as the sum of a set of effect matrices. The scores plots from the three submatrices (location, tester, trait) of are given in Fig. 3. The presence of a factor effect (observed SSQs; represented by the red vertical line in Fig. 3) can be visualized by determining whether that red line is distinct from the null distribution. It can be seen that separation between growing location and tester was observed and that this was statistically significant $(p<0.001)$. By contrast, the scores plots obtained from the "GM" submatrix involving a three-way comparison of the GM-trait 

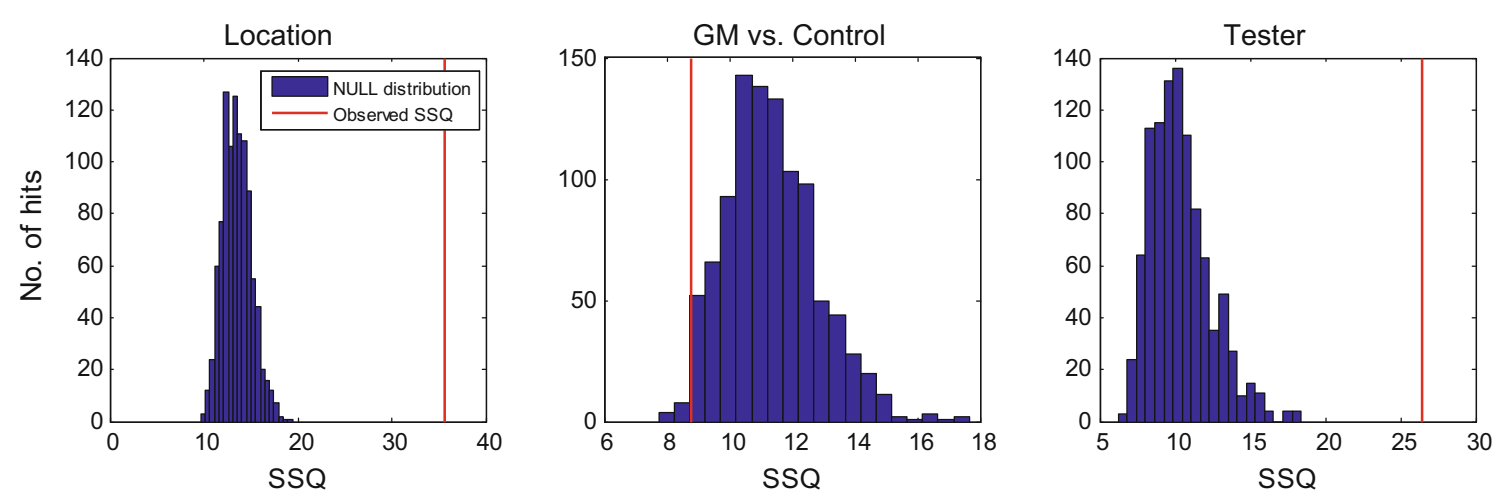

\begin{tabular}{|c|c|c|}
\hline Factor & Effect & p-values \\
\hline Location & $17.38 \%$ & $<0.001$ \\
\hline Trait & $0.90 \%$ & $>0.1$ \\
\hline Tester (S4062Y vs. T2052Z) & $5.26 \%$ & $<0.001$ \\
\hline Residual & $76.47 \%$ & - \\
\hline
\end{tabular}

Fig. 3 ASCA results showed the effects of location, trait, and tester. It can be seen that separation between growing location and tester was observed and $p$ values $<0.001$, for both factors, were obtained from the corresponding permutation tests. In other words, not a single case of 1000 permutations had obtained higher sum of squares (SSQs) than

positive, GM-trait-negative, and conventional hybrids showed no significant difference as seen in the figures of the observed SSQs superimposed on the corresponding null distribution. Indeed, the low value for the observed SSQs even in relation to the null distribution (Fig. 3) reiterates how negligible metabolomic differences between the GMtrait positive, GM-trait-negative, and conventional hybrids are. In summary, ASCA confirmed the results derived through MB-PCA that growing location was the factor with the most significant impact on the grain metabolome, followed by tester, whereas there was no significant differences between the GM and non-GM hybrid comparators.

The environmental influence on metabolic profiles of maize is consistent with that reported in other studies (Asiago et al. 2012; Baniasadi et al. 2014; Frank et al. 2012; Skogerson et al. 2010). The influence of the different testers on metabolomic variation is also consistent with studies on the influence of germplasm differences (same references) on the metabolome.

\subsection{Univariate analyses}

The main objective of the study was to evaluate the relative impact of "near-isogenic" effects in the context of comparative evaluations of GM and non-GM maize hybrids using MS-based metabolomics. We therefore also opted to the observed one. By contrast, the scores plots obtained from the GM submatrix involving a three-way comparison of the GM-trait positive, GM-trait-negative, and conventional hybrids, showed no significant difference as seen in the figures of the observed SSQs superimposed on the corresponding null distribution

pursue a univariate analysis extended to each identified metabolite individually. The univariate analysis approach followed that adopted in an earlier investigation of grain composition data (Venkatesh et al. 2015a). For all hybrids, least square mean values of each metabolite were determined across the three sites (termed a combined-site analysis). Subsequent analysis steps included, (i) statistical comparisons $(\alpha=0.05)$ of the combined-site mean for each grain metabolite a) between and within trait-positive and trait-negative hybrids, and b) between the conventional comparators and the trait-positive or trait-negative hybrids for each hybrid (tester) set, (ii) calculation of magnitudes of difference between the component individual site means of the trait-positive, trait-negative and control hybrids and the control component combined-site mean, and (iii) variance component analysis to assess the relative contributions to compositional variation of growing location, hybrid (tester) effect, and differences between the GM and non-GM comparators including those arising from residual genetic variation associated with back-crossing.

Supplementary Files S3 and S4 provide an overview of the combined-site means for the control and all positive and negative segregants for the S4062Y and T2502Z hybrid sets, respectively, as well as a summary of the univariate analyses. These results are discussed in more detail below. 


\subsubsection{Statistical comparisons}

For each metabolite, the following comparisons were conducted within each tester set (and shown in schematic form in Figures S2-S5). Firstly (Comparison 1, Figure S2), for each metabolite the combined-site mean of the control hybrid (i.e., the hybrid derived from the recurrent parent) was compared to the corresponding combined-site mean of each of the hybrids derived from each negative and positive segregant inbreds (Supplementary Files S3 and S4). No significant differences were observed for $96.32 \%$ and $96.08 \%$ of metabolite comparisons made, respectively (Table 2). Such results indicate that the trait-negative and trait-positive hybrids are not meaningfully different from the control and are consistent with an absence of any GM effect. Indeed, if the null hypothesis of a mean difference of zero were plausible, it would be reasonable to conclude that the observed differences simply reflect the number that would be observed by chance at the $5 \%$ significance level.

Secondly, (Comparison 2, Figure S3), the combined-site means for all metabolites of the trait-positive segregants were compared to the corresponding paired negative segregantderived hybrids (Fig. S1; Table 2). As shown in Table 2, there were no significant differences observed for $96.57 \%$ of the metabolite comparisons made. Consistent with the low number of significant differences, there were no analytes that were significantly different in all comparisons, i.e. no analytical trends distinguishing the traited and non-traited sets could be discerned (Tables S3, S4). In other words, differences between GM and non-GM comparators can arise even in stringent tests of paired trait-positive and trait-negative segregants but that the lack of reproducibility observed here implies that difference often may not reflect the effect of the GM trait. Further pairwise comparisons involving the trait-positive and traitnegative hybrids were conducted as described in detail in materials and methods. Thus, (Comparison 3, Figure S4), the combined-site means for each trait-positive hybrid were compared to each other (Supplementary Files S3 and S4); this pairwise scheme was then conducted for the trait-negative hybrids. Finally, (Comparison 4, Figure S5), in addition to the paired comparisons within Comparison 2, all remaining possible comparisons between trait-positive and trait-negative hybrids were made (Supplementary Files S2 and S3). As with Comparisons 1 and 2, results highlighted that, while statistically significant differences can arise in comparisons of paired trait-positive and trait-negative hybrids, their inconsistent expression suggests that factors other than the GM trait contribute.

\subsubsection{Effect size evaluation}

We hypothesized that, in this study, magnitudes of differences between the trait-positive, trait-negative, and corresponding conventional hybrids in levels of metabolites would be similar, and that the influence of GM, as well as of "near-isogenic" effects would be small, even if such magnitudes of differences could lead to findings of statistical significance. The context for evaluating magnitudes of differences was provided by an approach that involved expressing the individual site mean for each metabolite (whether from the trait-positive, trait-negative, or control) as the percent difference from the combined-site conventional control mean. In essence, any metabolite variation associated with the control hybrid comparisons would represent a location effect (i.e. differences due to growing location) only, whereas metabolite variation associated with the trait-positive and trait-negative hybrids would incorporate additional contributions from GM and "nearisogenic" effects.

Figure 4a shows, for all metabolites, the average percent difference from the combined-site conventional control mean, standard deviation, first percentile, and 99th percentile for the trait-positive, trait-negative and conventional hybrids (see Supplementary File S5 for results for individual metabolites). Figure $4 \mathrm{~b}$ provides information regarding the magnitudes of differences in histogram form. As is readily observed, the distribution and magnitudes of differences for metabolites assessed across each hybrid grouping are remarkably similar (see Supplementary File S6 for individual metabolites). This approach allows the distribution of magnitudes of differences observed for the trait-positive, trait-negative, and conventional hybrids to be visually compared to each other and shows that, broadly, the GM and "near-isogenic" effects assessed in this study have no major impact on metabolite variation.

\subsubsection{Variance components analysis}

Variance component analysis (VCA) was conducted to quantify the effect of the test factors on the maize grain metabolite profiles. The results of the VCA combined across all components is presented in Fig. 5 (see Supplementary File S7 for variation in levels of individual metabolites) and demonstrated that the contribution of near-isogenic and GM effects were extremely small relative to the much larger location and tester effects (Fig. 5). This is followed by a tester effect where the term "Tester" represents variation due to the two different females (testers) used in hybrid formation and the Rep (Location) effect. The term "Variant (Tester)" represents variation within the variants associated with a given tester and the term "Segregant (Variant Tester)" represents variation due to differences between the trait-positive and trait-negative segregants. These latter terms encompass variation that would be associated with any near-isogenic and GM trait effects. It should be note that although the term "Segregant 
Fig. 4 a (Upper table) shows the average percent difference (for all metabolites) from the combined-site conventional control mean, standard deviation, first percentile, and 99th percentile for the traitpositive, trait-negative and conventional control hybrids. b (Lower panels) presents the average percent difference from the combined-site conventional control mean in histogram form where the $\mathrm{x}$-axis is magnitude of difference and the $y$-axis is frequency of observations (expressed as percent)

\begin{tabular}{cccccc} 
A & \multicolumn{1}{l}{} & & \\
\hline Trait & Sample Size & $\begin{array}{c}\text { Average Percent } \\
\text { Difference }\end{array}$ & $\begin{array}{c}\text { Standard } \\
\text { Deviation }\end{array}$ & $\begin{array}{c}\text { 1st } \\
\text { Percentile }\end{array}$ & $\begin{array}{c}\text { 99th } \\
\text { Percentile }\end{array}$ \\
\hline Control & 918 & -0.00 & 24.67 & -65.16 & 61.91 \\
NEG & 3672 & -0.32 & 28.61 & -61.50 & 81.85 \\
POS & 3672 & 2.43 & 31.11 & -62.36 & 95.74 \\
\hline
\end{tabular}

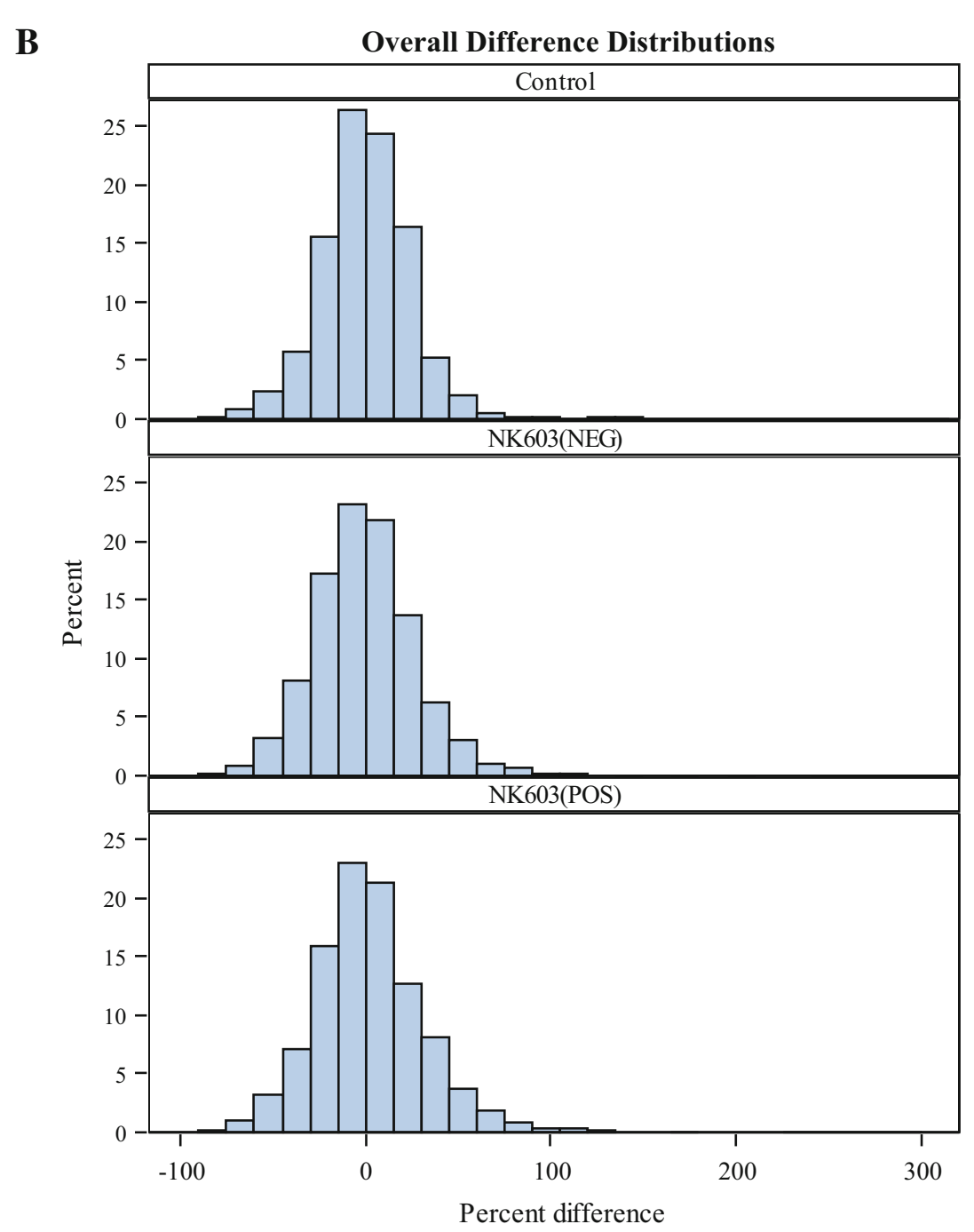

(Variant Tester)" involves a direct comparison of the POS and NEG variants (for each tester set) some contribution from a near-isogenic effect can be assumed and this term cannot strictly be viewed as a GM effect. Regardless, it is apparent that, overall, term "Segregant (Variant Tester)" is associated with the smallest source of variation in this study (Fig. 5). Indeed, this term was associated with precisely $0.0 \%$ variation for 113 of the 153 metabolites analyzed. Only a total of 6 metabolites (cholesterol, ethanolamine, lysine, melezitose, shikimic acid, and squalene) had a Segregant (Variant Tester) effect of $>5 \%$ (i.e. $147 / 153$ of metabolites had values $<5 \%$ ). Of these, most were associated with high residuals, and/or larger variation attributable to other factors such as location or tester, and iii) no consistent pattern of differences between the paired negative- and positive segregant-derived hybrids. As an illustrative example, lysine had a Segregant (Variant Tester) of $5.44 \%$ but the variance component term for location was $58.37 \%$. Pairwise comparisons of lysine levels showed that, for both the S4062Y and T2502Z hybrids 
Fig. 5 Variance component analysis averaged across all metabolites These results highlight the lack of any trait effect; the term Tester represents variation due to the two different female lines used in hybrid formation.

Location*Entry effect which represents the effect of the interaction between location and each of the hybrid entries. The term "Variant (Tester)" represents variation within the variants associated with a given tester and the term Segregant (Variant Tester) represents variation due to differences between the trait-positive and trait-negative segregants

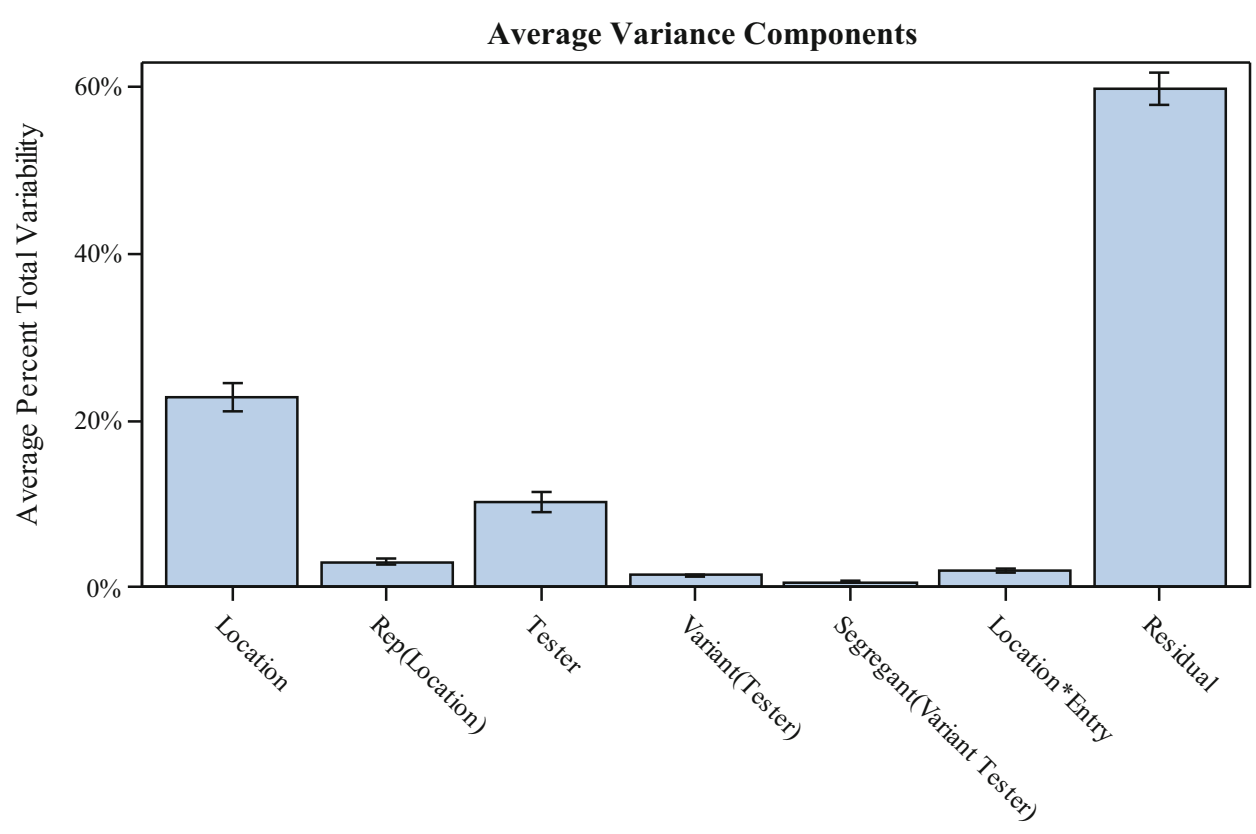

Variance Component

$\square$ Average Percent Total Variability, 1 Standard Error

tester, only one of the POS entries showed a statistically significant difference $(\alpha=0.05)$ when compared to the conventional control values. As an other example, differences in shikimic acid levels between POS and NEG segregants are associated with only one tester (S4062Y, Supplementary File S3) but not the other (T2502Z, Supplementary File S4).

These results, which highlight a lack of any consistent effect that could be associated with GM, are consistent with those of Venkatesh et al. $(2015 \mathrm{a}, \mathrm{b})$. The greater impact of germplasm and environment on compositional and metabolite variation is becoming increasingly wellestablished in studies on GM crops (Harrigan et al. 2010; Herman and Price 2013) and it is increasingly evident that the introduction of a GM trait is a negligible contributor to that variation, and even less than that associated with nearisogenic effects.

\section{Concluding remarks}

This study allowed a comparative evaluation of the effect of GM trait insertion and residual genetic variation on the maize grain metabolome in the context of variation associated with conventional breeding and growing location. Overall, the study was characterized by very few statistically significant differences and it was evident that GM trait insertion had little effect on the grain metabolome. Residual genetic variation was also only a minor contributor to variation but it was greater than that of the effect of
GM and could therefore be considered a potential source of statistically significant differences observed between GM and non-GM comparators. This was evidenced, in this study, by the fact that some differences observed between the GM trait-positive and trait-negative hybrids did arise even though these were never reproducible across the study for any metabolite assessed.

In an earlier publication (Venkatesh et al. 2015b), we evaluated maize grain composition in the context of natural variability associated with conventional germplasm and the impact of the multiple backcrossing steps used to develop both conventional and GM maize products. That study used the same sample set assessed here and the results showed that differences that would be observed in comparisons between any near-isogenic comparators, conventional or GM, can exceed that of GM effects but that the effect of GM and residual genetic variation on composition are markedly less than the effects of conventional breeding or growing location. The small number of statistically significant, but not biologically relevant, differences observed in composition studies of GM crops may, in most cases, simply be associated with residual genetic variation. The results from the current study described here show that this conclusion extends to assessments of the metabolome.

Our results have implications for practices and principles of safety assessments of GM crops. The absence of unintended biologically relevant compositional consequences observed after decades of composition studies on a range of GM crops support the safety of the GM process (Herman and Price 2013). Furthermore, in light of our 
results, the results of previous studies, whether compositional or based on omics technologies, that may have attributed minor differences between near-isogenic conventional and GM comparators to the presence of the GM trait may need to be re-interpreted. Metabolomics studies can only have merit in comparative assessment of GM crops if there is a hypothesis that directly associates changes in metabolite levels with both a given GM trait and with a potential safety concern. This is an unlikely scenario given the extensive variability of the grain metabolome attributable to factors considered to be associated with a history of safe use (e.g. differences in growing environment, conventional breeding) and the limited nutritional coverage of the metabolome particularly compared to that offered by traditional compositional studies. Issues related to safety and nutrition are therefore more effectively addressed through targeted hypothesis-driven evaluations based on compositional assessments that cover key nutrients and that provide clear interpretable endpoints.

Acknowledgments RG would like to thank the UK BBSRC for support in plant metabolomics (BB/J004103/1).

\section{Compliance with ethical standards}

Conflicts of Interest Several authors of this manuscript are or were employees of the Monsanto Company, one of the funders of this study.

Ethical Statement The biological materials used are of plant origin and so this article does not contain any studies with human participants or animals performed by any of the authors.

\section{References}

Asiago, V. M., Hazebroek, J., Harp, T., \& Zhong, C. (2012). Effects of genetics and environment on the metabolome of commercial maize hybrids: A multisite study. Journal of Agricultural and Food Chemistry, 60, 11498-11508.

Baniasadi, H., Vlahakis, C., Hazebroek, J., Zhong, C., \& Asiago, V. M. (2014). Effect of environment and genotype on commercial maize hybrids using LC/MS-based metabolomics. Journal of Agricultural and Food Chemistry, 62, 1412-1422.

Berman, K. H., Harrigan, G. G., Nemeth, M. A., Oliveira, W. S., Berger, G. U., \& Tagliaferro, F. S. (2011). Compositional equivalence of insect-protected glyphosate-tolerant soybean MON $87701 \times$ MON 89788 to conventional soybean extends across different world regions and multiple growing seasons. Journal of Agricultural and Food Chemistry, 59, 11643-11651.

Codex. (2009). Guideline for the conduct of food safety assessment of foods derived from recombinant DNA plants. CAC/GL 45-2003. Codex Alimentarius.

Davies, H. (2010). A role for "omics" technologies in food safety assessment. Food Control, 21, 1601-1610.

Eathington, S. R., Crosbie, T. M., Edwards, M. D., Reiter, R. S., \& Bull, J. K. (2007). Molecular markers in a commercial breeding program. Crop Science, 47, S154-S163.
EU. (2010). A decade of EU-funded GMO research 2001-2010. Directorate-General for Research and Innovation Biotechnologies, Agriculture, Food EUR 24473 EN.

Fiehn, O., Wohlgemuth, G., Scholz, M., Kind, T., Lee, D. Y., Lu, Y., et al. (2008). Quality control for plant metabolomics: Reporting MSI-compliant studies. The Plant Journal, 53, 691-704.

Frank, T., Röhlig, R. M., Davies, H. V., Barros, E., \& Engel, K. H. (2012). Metabolite profiling of maize kernels: Genetic modification versus environmental influence. Journal of Agricultural and Food Chemistry, 60, 3005-3012.

Goodacre, R., Vaidyanathan, S., Dunn, W. R., Harrigan, G. G., \& Kell, D. B. (2004). Metabolomics by numbers-Acquiring and understanding global metabolite data. Trends in Biotechnology, $22,245-252$.

Harrigan, G. G., \& Harrison, J. M. (2012). Assessing compositional variability through graphical analysis and Bayesian statistical approaches: case studies on transgenic crops. Biotechnology and Genetic Engineering Reviews, 28, 15-32.

Harrigan, G. G., Lundry, D., Drury, S., Berman, K., Riordan, S. G., Nemeth, M. A., et al. (2010). Natural variation in crop composition and the impact of transgenesis. Nature Biotechnology, 28, 402-404.

Herman, R. A., \& Price, W. D. (2013). Unintended compositional changes in genetically modified (GM) crops: 20 years of research. Journal of Agricultural and Food Chemistry, 61, 11695-11701.

James, C. (2014). Global status of commercialized biotech/GM crops: 2014. ISAAA Brief No. 49. International Service for the Acquisition of Agri-biotech Application: Ithaca, NY.

Kind, T., Wohlgemuth, G., Lee, D. Y., Lu, Y., Palazoglu, M., Shahbaz, S., et al. (2009). FiehnLib: Mass spectral and retention index libraries for metabolomics based on quadrupole and timeof-flight gas chromatography/mass spectrometry. Analytical Chemistry, 81, 10038-10048.

Ladics, G. S., Bartholomaeus, A., Bregitzer, P., Doerrer, N. G., Gray, A., \& Holzhauser, T. (2015). Genetic basis and detection of unintended effects in genetically modified crop plants. Transgenic Research, 24, 587-603.

OECD. (2006). An introduction to the food/feed safety consensus documents of the task force. Paris: Organization for Economic Cooperation and Development.

Prado, J. R., Segers, G., Voelker, T., Carson, D., Dobert, R., Phillips, J., et al. (2014). Genetically engineered crops: From idea to product. Annual Review of Plant Biology, 65, 769-790.

Privalle, L. S., Gillikin, N., \& Wandelt, C. (2013). Bringing a transgenic crop to market: Where compositional analysis fits. Journal of Agricultural and Food Chemistry, 61, 8260-8266.

Ricroch, A. E. (2013). Assessment of GE food safety using '-omics' techniques and long-term animal feeding studies. New Biotechnology, 30, 349-354.

Ricroch, A. E., Bergé, J. B., \& Kuntz, M. (2011). Evaluation of genetically engineered crops using transcriptomic, proteomic, and metabolomic profiling techniques. Plant Physiology, 155, $1752-1761$.

Rischer, H., \& Oksman-Caldentey, K. M. (2006). Unintended effects in genetically modified crops: Revealed by metabolomics. Trends in Biotechnology, 24, 102-104.

Röhlig, R. M., Eder, J., \& Engel, K. (2009). Metabolite profiling of maize grain: Differentiation due to genetics and environment. Metabolomics, 5, 459-477.

Schnell, J., Steele, M., Bean, J., Neuspiel, G. C., Dormann, N., et al. (2015). A comparative analysis of insertional effects in genetically engineered plants: Considerations for pre-market assessments Transgenic Research, 24, 1-17.

Skogerson, K., Harrigan, G. G., Reynolds, T. L., Halls, S. C., Ruebelt, M., Iandolino, A., et al. (2010). Impact of genetics 
and environment on the metabolite composition of maize grain. Journal of Agricultural and Food Chemistry, 58, 3600-3610.

Smilde, A. K., Jansen, J. J., Hoefsloot, H. C. J., Lamers, R.-J. A. N., van der Greef, J., \& Timmerman, M. E. (2005). ANOVAsimultaneous component analysis (ASCA): A new tool for analyzing designed metabolomics data. Bioinformatics, 21, 3043-3048.

Smilde, A. K., Westerhuis, J. A., \& de Jong, S. (2003). A framework for sequential multiblock component methods. Journal of Chemometrics, 17, 323-337.

Sumner, L. W., Amberg, A., Barrett, D., Beger, R., Beale, M. H., Daykin, C., et al. (2007). Proposed minimum reporting standards for chemical analysis. Metabolomics, 3, 211-221.

Venkatesh, T.V., Bell, E., Bicke1, A., Cook, K., Alsop, B., van de Mortel, M., et al. (2015b). Maize hybrids derived from GM positive and negative segregant inbreds are compositionally equivalent: any observed differences are associated with conventional backcrossing practices. Transgenic Research, accepted for publication, now online.

Venkatesh, T. V., Cook, K., Liu, B., Perez, T., Willse, A., Tichich, R., et al. (2015a). Compositional differences between near-isogenic
GM and conventional maize hybrids are associated with backcrossing practices in conventional breeding. Plant Biotechnology Journal, 13, 200-210.

$\mathrm{Xu}$, Y., \& Goodacre, R. (2012). Multiblock principal component analysis: an efficient tool for analyzing metabolomics data which contain two influential factors. Metabolomics, 8, 37-51.

$\mathrm{Xu}$, Y., Goodacre, R., \& Harrigan, G. G. (2014). Compositional equivalence of grain from multi-trait drought-tolerant miaze hybrids to a conventional comparator: Univariate and multivariate assessments. Journal of Agricultural and Food Chemistry, 62, 9597-9698.

Zhou, J., Harrigan, G. G., Berman, K. H., Webb, E. G., Klusmeyer, T. H., \& Nemeth, M. A. (2011). Stability in the composition equivalence of grain from insect-protected maize and seed from glyphosate-tolerant soybean to conventional counterparts over multiple seasons, locations, and breeding germplasms. Journal of Agricultural and Food Chemistry, 59, 8822-8828.

Zwanenburg, G., Hoefsloot, H. C. J., Westerhuis, J. A., Jansen, J. J., \& Smilde, A. K. (2011). ANOVA-principal component analysis and ANOVA-simultaneous component analysis: A comparison. Journal of Chemometrics, 25, 561-567. 